\title{
1 Lab-on-Chip for Testing Myelotoxic Effect of \\ 2 Drugs and Chemicals
}

6 Marco Rasponi

7 Department of Electronics, Information and Bioengineering, Politecnico di

8 Milano, Piazza Leonardo da Vinci 32, 20133 Milano, Italy.

$9+39(02) 2399-3377$

$10+39(02) 2399-3360$

11 marco.rasponi@polimi.it

12

13 Andrea Gazaneo

14 Department of Electronics, Information and Bioengineering, Politecnico di 15 Milano, Piazza Leonardo da Vinci 32, 20133 Milano, Italy.

16 andrea.gazaneo@polimi.it

18 Arianna Bonomi

19 Department of Biomedical, Surgical and Dental Sciences, Università degli Studi 20 di Milano, 20133 Milano, Italy.

21 arianna.bonomi@unimi.it

22

23 Andrea Ghiglietti

24 Department of Mathematics "Francesco Brioschi", Politecnico di Milano, 20133

25 Milano, Italy.

26 andrea.ghiglietti@polimi.it

28 Paola Occhetta

29 Department of Electronics, Information and Bioengineering, Politecnico di

30 Milano, Piazza Leonardo da Vinci 32, 20133 Milano, Italy.

31 paola.occhetta@polimi.it 
1 Gianfranco Beniamino Fiore

2 Department of Electronics, Information and Bioengineering, Politecnico di

3 Milano, Piazza Leonardo da Vinci 32, 20133 Milano, Italy.

4 gianfranco.fiore@polimi.it

5

6 Augusto Pessina

7 Department of Biomedical, Surgical and Dental Sciences, Università degli Studi

8 di Milano, 20133 Milano, Italy.

9 augusto.pessina@unimi.it

10

11 Alberto Redaelli*

12 Department of Electronics, Information and Bioengineering, Politecnico di

13 Milano, Piazza Leonardo da Vinci 32, 20133 Milano, Italy.

$14+39(02) 2399-3375$

$15+39(02) 2399-3360$

16 alberto.redaelli@polimi.it

17

18

$19 * \quad$ Corresponding author

20

21

22 


\section{Abstract}

2 In the last twenty years, one of the main goals in the drug discovery field has been the

3 development of reliable in vitro models. In particular, in 2006 the European Centre for the

4 Validation of Alternative Methods (ECVAM) has approved the Colony forming Unit-

5 Granulocytes-Macrophages (CFU-GM) test, which is the first and currently unique test applied to

6 evaluate the myelotoxicity of xenobiotics in vitro. The present work aimed at miniaturizing this in

7 vitro assay by developing and validating a Lab-on-Chip (LoC) platform consisting of a high

8 number of bioreactor chambers with screening capabilities in a high-throughput regime.

10 Keywords

11 Lab-on-Chip

12 Microfluidics

13 Clonogenic test

$14 C F U-G M$

15 CBMNC

16

17 


\section{Introduction}

2 In the last twenty years, one of the main goals in the drug discovery field has been

3 the development of reliable in vitro models. Being currently the in vivo testing

4 phase still necessary - to approve new drugs it is necessary to perform in vivo

5 studies including at least two animal species, and employing various dosing

6 schedules, depending on the molecule to be approved (Boorman et al. 1982) -

7 research has been focusing on the development of new platforms able to replace,

8 or reduce, the traditional in vivo trials at least in the early stages of the drug

9 discovery process. Advantages of the in vitro approach mainly rely on economical

10 and ethical standpoints. Indeed, in vitro testing presents numerous advantages,

11 namely the reduction of model-to-model variability, ease of data collection, less

12 requirements in terms of personnel training, reduction in the amounts of reagents,

13 miniaturization and automation (Hartung and Daston 2009).

14 One of the fields which could mainly benefit from the paradigm mutation towards

15 in vitro experimentation is toxicology, i.e. the study of drug toxicity (Gad 1990).

16 In particular, clonogenic assays are thought to better bridge the gap between

17 toxicity experienced in vivo and toxicity effects on cells cultivated in laboratory

18 (cytotoxicity). Clonogenic assays are cytotoxicity tests based on the rate of

19 survival of colony forming units (CFUs) in the presence of several drug

20 concentrations, thus differing from traditional proliferation assays which take into

21 account the mere increase in cell number. Specific advantages of clonogenic

22 assays are related to higher accuracy and predictivity with respect to traditional

23 proliferation tests (Pessina et al. 2004).

24 To date, the European Centre for Validation of Alternative Methods (ECVAM)

25 has approved only one clonogenic test (DB-ALM protocol 101, available on

$26 \mathrm{http} / /$ ecvam-dbalm.jrc.ec.europa.eu/). This particular test aims at characterizing

27 the acute effect of toxicants on granulocytes and macrophages (GM) progenitor

28 cells derived both from bone marrow and umbilical cord blood, under maximally

29 stimulatory cytokine concentrations. The system is based on counting CFUs

30 formed by the surviving progenitors after xenobiotics exposure.

31 In 2004, Pessina and co-workers optimized a new protocol for CFU-GM assay

32 based on the use of 96-well plates in place of standard Petri dishes thus reducing

33 complexity and costs of the original test. 
1 However, the assay still remains time-consuming. Colonies are generally counted

2 only upon 14 days of incubation. In addition, the assay implies the dispersion of

3 cells within a semisolid culture medium, which inhibits a pre-determined

4 localization of colonies (within dishes or wells), which all must be manually

5 found by a trained operator (Pessina et al. 2001).

6 Our goal was to develop a microfluidic device able to miniaturize the current

7 state-of-art paradigm of in vitro hematotoxicity of xenobiotics protocol. Indeed, a

8 further miniaturization of the original assay, down to the micrometer scale, will

9 provide at the same time advantages in terms of cost reduction (cells, drugs,

10 cytokines, etc.) and decrease of testing time (colony forming process). To this aim

11 we designed a novel Lab-on-Chip (LoC) platform enabling for trapping, feeding

12 and growing of multipotent monoclonal stem cells.

\section{Materials and Methods}

\section{$14 \quad 2.1 \quad$ Lab-on-Chip design and fabrication}

15 A grid-like distribution of micro-chambers was envisaged within the LoC device

16 to organize the spatial distribution of cells and, in turn, to obtain a univocal

17 correspondence between colonies and culture chambers.

18 For this purpose, we designed a microfluidic platform divided in two functional

19 areas: a serial dilution generator (Jeon et al. 2002; Kim et al. 2008; Lee et al.

20 2009; Occhetta et al. 2015a) and a culture region (Fig. 1). The first one consists of

21 a fluidic network of channels ( $70 \mu \mathrm{m}$ high and $70 \mu \mathrm{m}$ wide) arranged in a resistive

22 flow-based scheme to deliver six linear dilutions of drugs (concentration ranging

23 from 1 to 0 , step 0.2 ) from two input ports to the culture region. This area was

24 integrated envisioning the possibility to test the cytotoxic effect of different dose

25 of drugs on bone marrow progenitor cells within a single device.

26 Downstream, the culture region consists of 180 cubic chambers (side $150 \mu \mathrm{m}$ )

27 divided in 6 units, each composed by 3 parallel lines of ten culture chambers.

28 The microfluidic device is made of polydimethylsiloxane (PDMS) and fabricated

29 by means of standard soft lithography techniques. Briefly, a 4" silicon wafer,

30 microstructured with two photoresist types, served as master mold to cast a thin

31 layer (about $1 \mathrm{~mm}$ ) of liquid PDMS, being curing agent and pre-polymer mixed in

32 a ratio $1: 10(\mathrm{w} / \mathrm{w})$. A 3 hours thermal treatment at $65^{\circ} \mathrm{C}$ was applied to achieve a 
1 complete crosslinking. Subsequently, the cured PDMS stamp was removed from

2 the mold and bonded, upon an air plasma treatment, to a previously casted slab of

3 PDMS (thickness $4 \mathrm{~mm}$ ) to close the fluidic features. The assembly was trimmed

4 and drilled in correspondence of 4 inputs and 6 outputs, by means of biopsy

5 punchers having diameters of 0.5 and $3 \mathrm{~mm}$, respectively. Finally, the LoC device

6 was bonded to a histology glass slide upon a further air plasma treatment,

7 sterilized through autoclaving $\left(121^{\circ} \mathrm{C}, 20 \mathrm{~min}\right.$, wet cycle $)$ and subsequently dried

8 overnight at $80^{\circ} \mathrm{C}$ to recover PDMS hydrophobicity after plasma treatment, thus

9 minimizing cell adhesion.

11 Fig. 1 The layout of the Lab-on-Chip device is shown together with a detail of a group of

12 chambers within a culture unit. Two main input ports (together with two secondary ports) deliver

13 dilutions of chemicals (100\%,80\%,60\%,40\%, 20\%, CTRL) to 6 culture units (30 chambers each)

\section{$14 \quad 2.2$ Cell expansion and characterization}

15 As a source of stem cells, the mononucleated cell fraction from human umbilical

16 cord blood (CB-MNCs) purchased frozen from Lonza (Swiss) was used. The

17 cells, stored in liquid nitrogen until use, were thawed according to a previously

18 described standard operating procedure (Pessina et al., 2004). Briefly, thawed

19 cells were transferred from the cryovial to a $15 \mathrm{ml}$ conical tube and swirled while

20 warmed thawing medium (Iscove's Modified Dulbecco Medium (IMDM)

21 supplemented with 10\% Fetal Bovine Serum (FBS) and $10 \mathrm{U} / \mathrm{ml}$ Dnase I) was

22 added drop by drop to the cells. The cell suspension was then centrifuged at $200 \mathrm{~g}$

23 for $15 \mathrm{~min}$ at room temperature. 
1 Most of the wash was removed by pipette leaving 1-2 $\mathrm{ml}$ behind so the cell pellet

2 was not disturbed and was then resuspended in the remaining medium. Fresh

3 IMDM with 1\% l-glutamine and 10\% FBS was added to the cell suspension and

4 then centrifuged as above described. All the reagents were purchased from

5 EuroClone (Italy). Cells were then resuspended in Complete Medium (CM),

6 consisting of IMDM, containing 30\% FBS, 1\% L-Glutamine, 1\%

7 Penicillin/Streptomycin solution and $10 \mathrm{ng} / \mathrm{ml}$ recombinant human granulocyte/

8 macrophage colony-stimulating factor ( $\mathrm{r}$ hu GM-CSF, RELIATech, Germany) to

9 evaluate their number and viability.

10 Subsequently, CFU based tests were performed by using pooled donor cord

11 blood-derived CD34+ cells, purchased frozen from Life Technologies, USA. The

12 cells were stored and thawed according to the same protocol applied for CB-

13 MNCs.

\section{$14 \quad 2.3 \quad$ Cell seeding within the LoC platform}

15 Preliminary tests were carried out to establish a correlation between cell seeding

16 conditions and number of cells trapped in each chamber. For this purpose CB

17 MNCs were used. Aliquots of $30 \mu 1$ of cell suspension (concentration of 500,000

18 cells $/ \mathrm{ml}$ ) were simultaneously injected into both input ports through a dual

19 channel syringe pump at a flow rate of either 0.1 or $0.2 \mu \mathrm{l} / \mathrm{min}$. Upon complete

20 seeding, a picture of each chamber was taken through a phase contrast

21 microscope, and the number of cells contained in each chamber was directly

22 quantified. For these experiments, a number of 9 and 8 replicates ( $n=9$ and $n=8)$

23 were used for 0.1 and $0.2 \mu 1 / \mathrm{min}$, respectively.

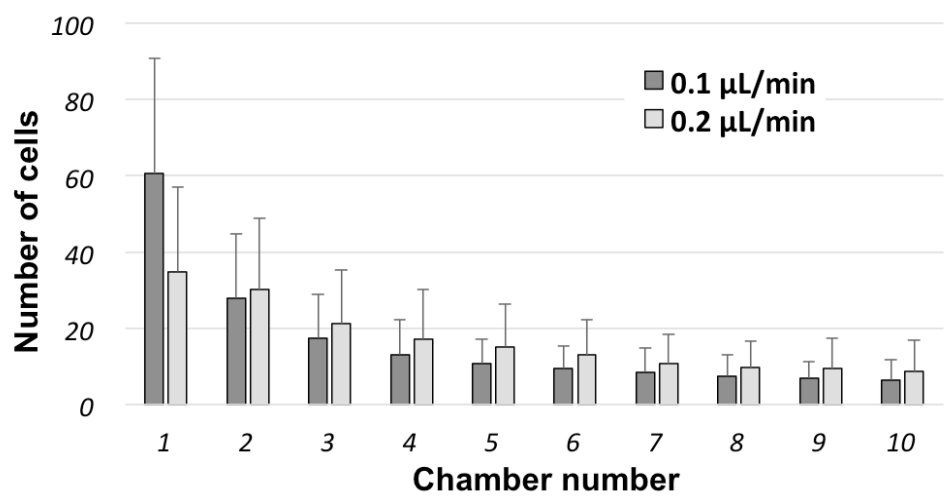

26 Fig. 2. Content in terms of cell number for each of the 10 chambers encountered by cells in their 27 stream. Each device has 18 decades of chambers, and experiments were carried out for 9 and 8 
replicates for 0.1 and $0.2 \mu 1 /$ min flow rates, respectively. Data is presented as mean \pm standard

deviation

4 The cell distribution for both conditions tested is depicted in Fig. 2, which shows

5 a decrement in population along chambers in the same fluidic path. In particular,

6 the number of cells present in each chamber at the end of the seeding process

7 resulted $14.2 \pm 26.2$ and $16.8 \pm 18.7$ for flow rates of 0.1 and $0.2 \mu 1 / \mathrm{min}$,

8 respectively. Although these values were similar, the higher flow rate provided a

9 significantly lower number of empty chambers (24.4 \pm 22.8 vs $11.4 \pm 9.5)$, while

10 reducing the seeding time ( 2.5 vs 5 hours). Thus $0.2 \mu 1 / \mathrm{min}$ was chosen as seeding

11 condition for the following experiments and corresponding data were used in the

12 statistical model. Immediately after seeding, CB MNCs viability within the device

13 was estimated to be higher than $90 \%$ (see Supplementary Information).

\section{$14 \quad 2.4 \quad$ Statistical model of the clonogenic assay}

15 In general, the toxic effect of a drug molecule tested in a clonogenic assay results

16 in a smaller number of CFUs with respect to a control condition, wherein no drug

17 is administered. For a suitable statistical analysis, we modeled this situation as if

18 the drug was able to decrease the probability of any single cell to be clonogenic,

19 while keeping the number of cells constant.

20 We denote with $\mathbf{p}_{\mathbf{0}}$ the probability of any cell to form a colony in the control. This

21 value does not depend on the chamber volume and it is also independent of time,

22 thus it is an intrinsic property of the cell donor. According to the results obtained

23 with standard macroscale experiments, in our case study $\mathbf{p}_{\mathbf{0}}$ was set equal to

$24 \quad 1 / 146$. We then set a hypothesis test to determine if there is statistical evidence to

25 state whether the probability $\mathbf{p}$ of a cell to form a colony when drug is

26 administered is smaller than the probability $\mathbf{p}_{\mathbf{0}}$ in the control. Hence, in the null

27 hypothesis we have $\mathbf{p}=\mathbf{p}_{0}$, while in the alternative hypothesis we have $\mathbf{p}=\mathbf{p}_{\mathbf{1}}<\mathbf{p}_{\mathbf{0}}$.

28 The seeding process suggested us to model the number of cells injected in each

29 chamber as a random variable with a probability distribution depending on the

30 position of the chamber in the corresponding line. Moreover, the numbers of cells

31 in the chambers were treated as independent variables, and all the lines and units

32 were considered to have the same distribution of cells. From the data collected in

33 the seeding characterization tests (Fig. 2, perfusion rate $0.2 \mu 1 / \mathrm{min}$ ) we computed 
1 the empirical distribution of the number of cells for each position within the

2 culture line (constituted by 10 chambers).

3 The probability to find a colony in a chamber was obtained by modelling the

4 number of clonogenic cells per chamber with a binomial law, having size equal to

5 the average number of cell in that chamber and probability equal to $\mathbf{p}_{\mathbf{0}}$. Hence, the

6 number of positive chambers (chambers + , i.e. chambers containing at least one

7 colony) in a chip composed by $\mathbf{n}$ chambers ( $\mathbf{n} / \mathbf{1 0}$ culture lines) is defined by a

8 sum of $\mathbf{n}$ independent and identical distribution binomial random variables whose

9 parameters are those defined above. Since the probability distribution of this

10 random variable does not belong to any family of distributions known in statistics,

11 its density cannot be expressed analytically but it must be computed through

12 simulations. These simulations were run through the $\mathrm{R}$ environment (software

13 version 2.13.1), and a set of 10000 repetitions was enough to obtain stable

14 distributions.

15 A critical region for the hypothesis test was built as it follows: the null hypothesis

16 (i.e. a cell is clonogenic with a probability equal to $\mathbf{p}_{\mathbf{0}}$ ) has to be rejected

17 whenever the number of chambers+ in the chip is less than a threshold $\mathbf{C}$. The

18 threshold value depends upon the significance level $\boldsymbol{\alpha}$ of the test and the number

19 of chambers in the chip $\mathbf{n}$. For instance, by setting $\boldsymbol{\alpha}=0.05$, in our chip $(\mathbf{n}=180)$ we

20 obtained $\mathbf{C}=12$.

21 Furthermore, we computed the power of the test for different values of $\mathbf{p}_{\mathbf{1}}$, that is

22 the probability of having less than $\mathbf{C}$ chambers + due to the effect of the

23 administered drug $\left(\mathbf{p}_{1}<\mathbf{p}_{0}\right)$. In Fig. 3, for instance, we show the probability

24 distribution of the chambers + for a particular choice of $\mathbf{p}_{\mathbf{1}}=1 / 2 \mathbf{p}_{\mathbf{0}}=1 / 292$,

25 corresponding to the ideal IC50 for a drug molecule. In this case, the power

26 resulted equal to $73.8 \%$.

27

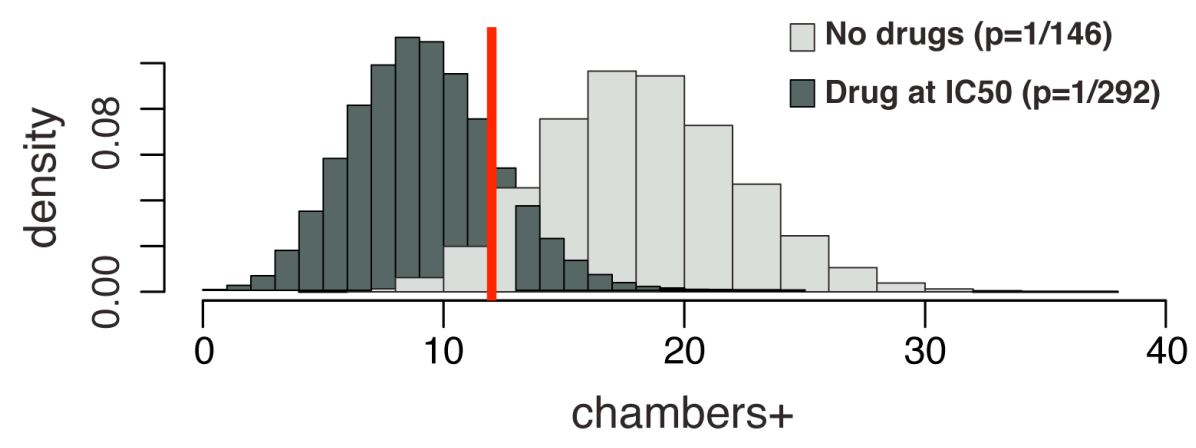


Fig. 3. Probability distributions of the number of chambers + within the chip both when no drug (light grey; probability of proliferation $\mathbf{p}_{\mathbf{0}}=1 / 146$ ) and a drug at a concentration corresponding to the IC50 (dark grey; $\mathbf{p}_{1}=1 / 292$ ) are administered. The vertical red line indicates the threshold

6 The power of the test computed above is only valid when the number of chambers

7 in the chip is equal to 180. In Fig. 4 we show how the power of the test depends

8 on $\mathbf{n}$. The vertical red dotted line represents the current chip with $\mathbf{n}=180$, whose

9 power is equal to 0.738 . The power rapidly approaches its maximum value when

10 the number of chambers increases (it exceeds 0.9 already with $\mathbf{n}=240$ ).

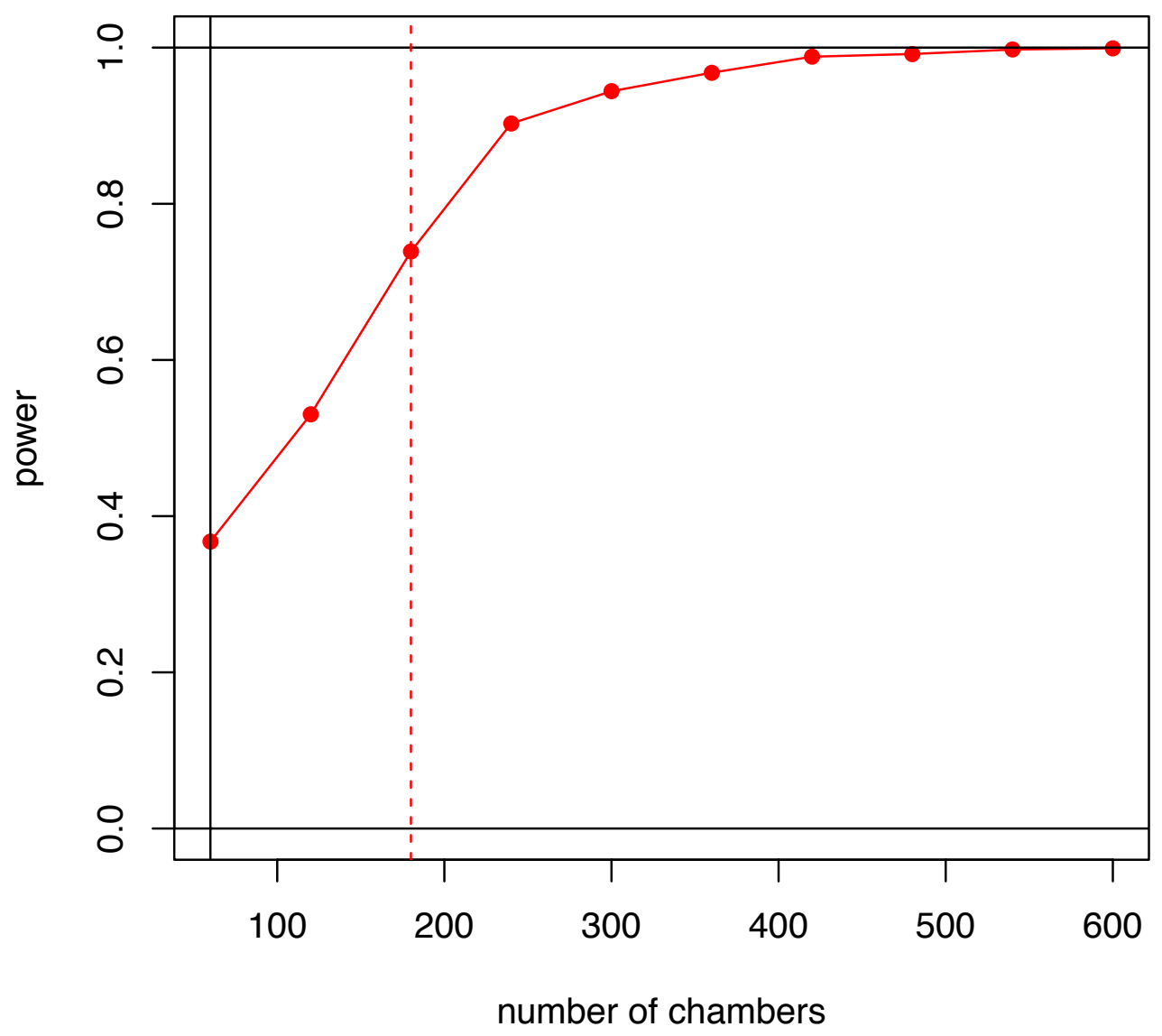

Fig. 4. Power of the test as a function of the number of chambers in the chip (n). The vertical red dotted line represents the current chip with $\mathbf{n}=180$, whose power is equal to 0.738 .

\section{$14 \quad 2.5 \quad$ Implementation of the microscale clonogenic assay}

$15 \mathrm{CD} 34+$ cells were seeded into the LoC device at a flow rate of $0.2 \mu \mathrm{l} / \mathrm{min}$. Upon

162.5 hours, $\mathrm{CM}$ was prepared and perfused into the device at the same flow rate 
1 throughout the entire culture period (14 days), according to previous results in a

2 similar platform (Occhetta et al. 2015b). At specific time points (days 1, 5, 7 and

3 14) pictures from all culture chambers of the LoC device were taken to monitor

4 possible variations in cell number. The results were compared to control

5 experiments, consisting of devices perfused with culture medium free from

6 cytokines.

7 CFU potential of CD34+ cells within the microfluidic device was also assessed

8 both in the absence and presence of a drug with a recognized hematotoxicity in

9 clinical use. In particular, $1200 \mathrm{ng} / \mathrm{ml}$ (200X) of Paclitaxel (Toronto Research

10 Chemicals, Canada) dissolved in dimethylsulfoxide (DMSO, Serva, Germany),

11 were added to CM in order to have a final drug concentration of $6 \mathrm{ng} / \mathrm{ml}$ and a

12 DMSO percentage of $0.5 \%(\mathrm{v} / \mathrm{v})$. Paclitaxel was delivered to cells through the

13 input ports through continuous perfusion. The culture was carried out up to 7

14 days, and pictures of each chamber were taken at days 1,5 and 7. Pictures from

15 the control device were also taken at the same time-points and used as positive 16 control.

\section{Results and Discussion}

18 The 2-weeks culture period within the microfluidic platform provided an

19 increasing number of CD34+ cells in each chamber from day 1 to day 14 when

20 perfused with $\mathrm{CM}$ with cytokines, being the initial number of cells per chamber

$21 \quad 13.1 \pm 15.3$ and the final $24.4 \pm 21.0$. Conversely, the number of cells did not

22 change significantly along the culture period in the control experiment without

23 cytokines (with a percentage increase of only $0.57 \%$ ).

24 In particular, we noticed an increased in the number of cells from day 1 to day 5

$25(21.0 \pm 19.0)$, thus suggesting the possibility to reduce the assay time by

26 identifying the chamber containing colonies (positive chambers, chamber+)

27 within a shorter five days period of culture in vitro. 


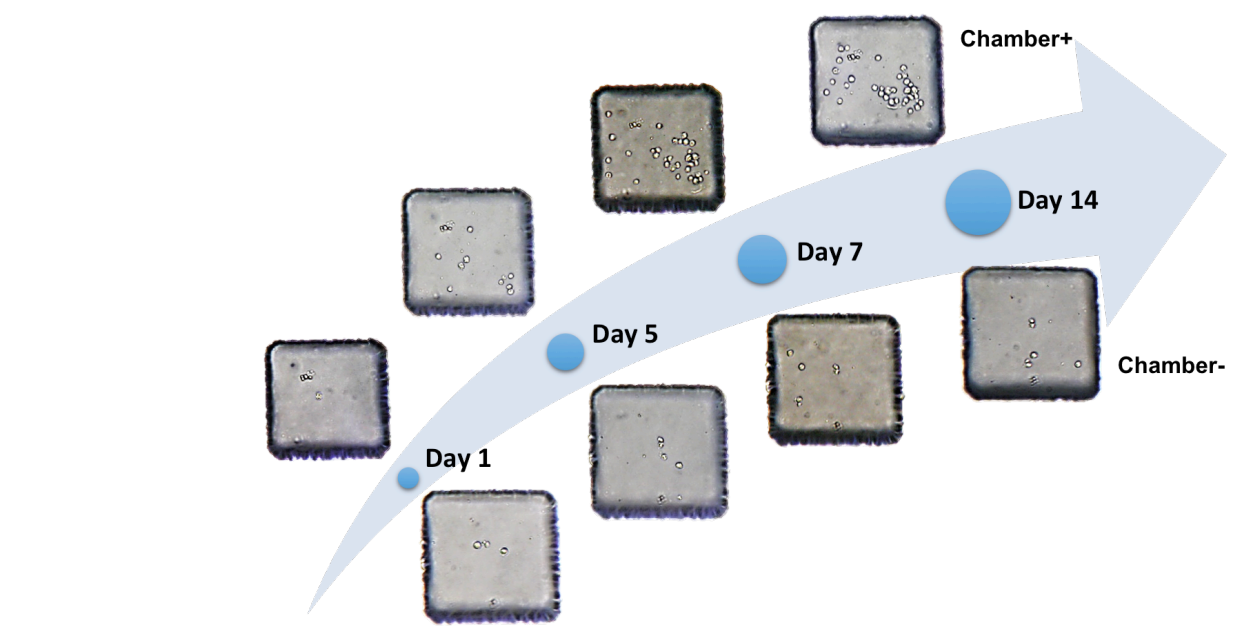

Fig. 5. Examples of a chamber containing at least one CFU (top) and no CFU (bottom)

4 The number of chamber+ was inferred from pictures for both conditions. Fig. 5

5 shows the content of two exemplifying chambers. In particular, a chamber was set

6 positive in case the following conditions were simultaneously true:

$8 \quad i . \quad$ relative cell number increase $>25 \%$;

9 ii. absolute cell number increase $>4$.

11 The number of resulting chamber+ was $15.33 \pm 6.11(n=3)$, in agreement with the

12 statistical model.

13 Furthermore, as preliminary test, the number of chamber+ was also assessed in

14 presence of Paclitaxel, a prototype toxicant molecule whose dosage was chosen,

15 in agreement with our previous findings corresponding to half maximal inhibitory

16 concentration (IC50). The number of chamber+ in absence of any toxicant

17 substances was 14 (positive control), while chamber+ number was reduced to 6

18 when the drug was delivered. Fig. 6 shows a graphical representation of the

19 distributions of chamber+ (green), chamber- (yellow) and empty chambers (red)

20 for both cases. 
CONTROL

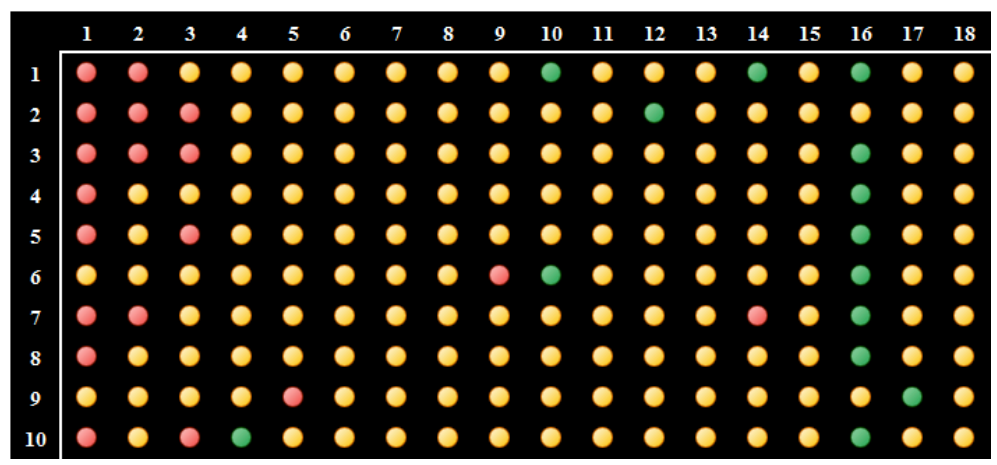

DMSO \& DRUG

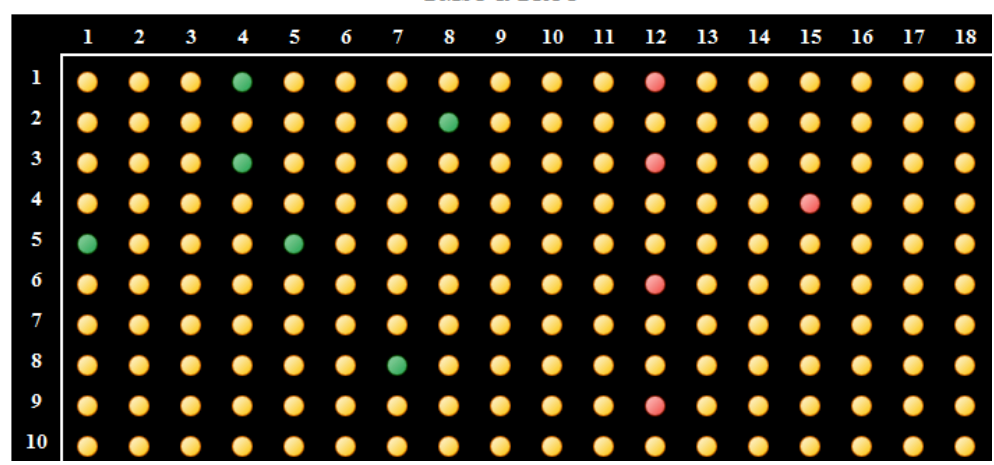

Fig. 6. Representation of positive (green), negative (yellow) and empty (red) chambers

4 This result suggests that the device, together with the implemented statistical

5 model, allows identifying the toxicity of a test molecule at a specific

6 concentration.

\section{Conclusions}

8 The proposed LoC device demonstrated its suitability as platform for CFU-based

9 assays. The average number of colonies formed after five days of culture (15.33 \pm

10 6.11) was adequate to detect the presence of a toxic dosage (IC50) of a drug. The

11 assay time was dramatically reduced in two ways: i) the culture time decreased

12 from 14 to 5 days, and ii) the colony identification was reduced to microscopy

13 acquisitions on a well-defined plane (chamber/channel fluidic layer).

14 The device was provided with a serial dilution generator unit, able to precisely

15 deliver to culture units predetermined concentration of soluble factors. However,

16 the number of chambers implemented for these experiments $(n=180)$ was only

17 enough to catch the effect of a single test condition - namely the concentration of

18 the administered molecule - and this unit was not used. Neverthless, by simply

19 increasing the number of chambers contained in each culture unit (e.g. to 180 or

20 more), the device could be exploited to fully implement a miniaturized clonogenic 
test directly on-chip. As described, the increase of the number of culture chambers

2 is also expected to improve the power of the statistical model; indeed, finer cutoff

3 thresholds could be considered to exclude chambers initially containing a number

4 of cells statistically different from the expected one.

5 Remarkably, a straightforward integration of this miniaturized model with a real-

6 time acquisition system for the automatic detection and quantification of colonies,

7 easily feasible thanks to the pre-determined and ordered positions of CFUs within

8 the optically transparent device, would represent a huge step towards an automatic

9 and user-friendly implementation of the clonogenic test.

\section{Acknowledgements}

This work was financially supported by Fondazione Cariplo, grant no. 2011-0322.

\section{References}

Boorman G, Luster M, Dean J, Campbell M (1982) Assessment of myelotoxicity caused by environmental chemicals Environmental health perspectives 43:129

Gad SC (1990) Recent developments in replacing, reducing, and refining animal use in toxicologic research and testing Toxicological sciences 15:8-16

Hartung T, Daston G (2009) Are in vitro tests suitable for regulatory use? Toxicological sciences 111:233-237

Jeon NL, Baskaran H, Dertinger SK, Whitesides GM, Van De Water L, Toner M (2002) Neutrophil chemotaxis in linear and complex gradients of interleukin- 8 formed in a microfabricated device Nature biotechnology 20:826-830

Kim C, Lee K, Kim JH, Shin KS, Lee K-J, Kim TS, Kang JY (2008) A serial dilution microfluidic device using a ladder network generating logarithmic or linear concentrations Lab on a Chip 8:473-479

Lee K et al. (2009) Generalized serial dilution module for monotonic and arbitrary microfluidic gradient generators Lab on a Chip 9:709-717

Occhetta P et al. (2015) High-throughput microfluidic platform for adherent single cells non-viral gene delivery RSC Advances 5:5087-5095

Occhetta $\mathrm{P}$ et al. (2015) High-throughput microfluidic platform for adherent single cells non-viral gene delivery Reports 5:10288

Pessina A et al. (2001) Prevalidation of a model for predicting acute neutropenia by colony forming unit granulocyte/macrophage (CFU-GM) assay Toxicology in vitro 15:729-740

Pessina A et al. (2004) A methylcellulose microculture assay for the in vitro assessment of drug toxicity on granulocyte/macrophage progenitors (CFUGM) Alternatives to laboratory animals: ATLA 32:17-23 